\title{
Feasibility, uptake and impact of a hospital-wide tobacco addiction treatment pathway: Results from the CURE project pilot
}

\author{
Authors: Matthew Evison, ${ }^{\mathrm{A}}$ Cheryl Pearse, ${ }^{\mathrm{B}}$ Freya Howle, ${ }^{\mathrm{C}}$ Monique Baugh, ${ }^{\mathrm{D}}$ Helen Huddart, ${ }^{\mathrm{D}}$ Eileen Ashton, ${ }^{\mathrm{D}}$ \\ Michael Rutherford, ${ }^{\mathrm{D}}$ Carol Kearney, ${ }^{\mathrm{D}}$ Lynn Elsey, ${ }^{\mathrm{E}}$ Darren Staniforth, ${ }^{\mathrm{E}}$ Kathryn Hoyle, ${ }^{\mathrm{F}}$ Murugesan Raja, ${ }^{\mathrm{G}}$ \\ Julie Jerram, ${ }^{\mathrm{H}}$ David Regan, ${ }^{\mathrm{I}}$ Richard Booton, ${ }^{\mathrm{A}}$ John Britton, ${ }^{\mathrm{C}}$ Claire O'Rourke, ${ }^{\mathrm{K}}$ David Shackley, ${ }^{\mathrm{L}}$ Liz Benbow, ${ }^{\mathrm{M}}$ \\ Andrea Crossfield, ${ }^{\mathrm{N}}$ Jayne Pilkington, ${ }^{\mathrm{O}}$ Mandy Bailey ${ }^{\mathrm{P}}$ and Richard Preece ${ }^{\mathrm{Q}}$
}

\section{Introduction}

Providing comprehensive tobacco addiction treatment to smokers admitted to acute care settings represents an opportunity to realise major health resource savings and population health improvements.

\section{Methods}

The CURE project is a hospital-wide tobacco addiction treatment service piloted in Wythenshawe Hospital, Manchester, UK. The core components of the project are electronic screening of all patients to identify smokers; the provision of brief advice and pharmacotherapy by frontline staff; opt-out referral of smokers to a specialist team for inpatient behavioural interventions; and continued support after discharge.

Authors: ${ }^{\text {A }}$ consultant in respiratory medicine and CURE clinical lead, Wythenshawe Hospital, Manchester, UK; ${ }^{\text {B lead CURE }}$ specialist nurse, Wythenshawe Hospital, Manchester, UK; ${ }^{C}$ CURE programme manager, Greater Manchester Cancer, Manchester, UK; ${ }^{D}$ CURE specialist nurse, Wythenshawe Hospital, Manchester, UK; Epharmacist, Wythenshawe Hospital, Manchester, UK; ${ }^{F}$ medicines optimisation pharmacist, Manchester Health \& Care Commissioning, Manchester, UK; ${ }^{G}$ general practitioner clinical lead for respiratory medicine, Manchester Health \& Care Commissioning, Manchester, UK; ${ }^{H}$ programme lead for the Living Well team, Manchester Health \& Care Commissioning, Manchester, UK; ' ${ }^{\mathrm{I}}$ director of population health, nursing and safeguarding, Manchester Health \& Care Commissioning, Manchester, UK; ${ }^{j}$ professor of epidemiology and consultant in respiratory medicine, Nottingham University, Nottingham, UK; ${ }^{\mathrm{K}}$ associate director, Greater Manchester Cancer, Manchester, UK; ' ${ }^{\text {medical }}$ director, Greater Manchester Cancer, Manchester, UK; ${ }^{\text {M }}$ strategic lead, Greater Manchester Health and Social Care Partnership, Manchester, UK; N population health policy and strategy specialist, Greater Manchester Health and Social Care Partnership, Manchester, UK; ${ }^{\circ}$ deputy director of population health, Greater Manchester Health and Social Care Partnership, Manchester, UK; ${ }^{P}$ chief executive, Wythenshawe Hospital, Manchester, UK; ${ }^{Q}$ director for quality, Greater Manchester Health and Social Care Partnership, Manchester, UK

\section{Results}

From 01 October 2018 to 31 March 2019, 92\% (13,515/14,690) of adult admissions were screened for smoking status, identifying 2,393 current smokers. Of these, $96 \%$ were given brief advice to quit by the admitting team. Through the automated 'opt-out' referral process, $61 \%$ patients completed inpatient behavioural interventions with a specialist cessation practitioner (69\% within the first 48 hours of admission). Overall, $66 \%$ of smokers were prescribed pharmacotherapy. Over one in five of all smokers admitted during this pilot reported that they were abstinent from smoking 12 weeks after discharge $(22 \%)$ at a cost $£ 183$ per quit.

\section{Discussion}

National implementation of this cost-effective programme would be likely to generate substantial benefits to public health.

KEYWORDS: Tobacco, smoking cessation, tobacco addiction

DOI: $10.7861 /$ clinmed.2019-0336

\section{Introduction}

Smoking tobacco is the single biggest cause of preventable death, disability, ill-health and social inequality in the UK. ${ }^{1}$ Treating tobacco addiction is one of the most cost-effective health interventions the NHS can provide, delivering additional life-years at $1 / 25$ of the cost of statin therapy in patients with coronary artery disease. ${ }^{2}$ Furthermore, smoking causes a significant burden to secondary care in the NHS, costing $£ 620$ million in England alone through hospital admissions in 2015/2016. However, a hospital admission represents a unique opportunity and 'teachable moment' to treat tobacco addiction, the substantial benefits of which were demonstrated in hospitalised smokers in the Ottawa Model for Smoking Cessation (OMSC), which reported a reduction in readmission rates from $38.4 \%$ to $26.7 \%$ and a halving of mortality from $11.4 \%$ to $5.4 \%$ at 12 months. ${ }^{3}$ If the same benefits could be replicated in the NHS, the resource savings and lives saved would be highly significant. ${ }^{1,4}$ However, current 
provision of smoking cessation interventions in UK hospitals has been described as 'woefully inadequate', with roughly $5 \%$ of smokers receiving either medications or specialist support during a typical hospital admission. ${ }^{5}$

There is, therefore, an urgent need to implement comprehensive hospital-wide tobacco addiction treatment services that provide all smokers with evidence-based interventions to stop smoking when they are admitted to hospital. Timely access to medications and specialist support must be the cornerstone of such services, provided as an 'opt-out' model with all healthcare professionals taking responsibility for their part in the service. ${ }^{1,6-9}$ We report the feasibility, uptake and impact of the first UK pilot study of such a service: the Conversation, Understand, Replace, Experts and evidence-based treatments (CURE) project in Manchester.

\section{Methods}

\section{Study outline}

The CURE project was a pilot study at Wythenshawe Hospital, part of Manchester University NHS Foundation Trust, in collaboration with the Greater Manchester Make Smoking History team and Greater Manchester Cancer (the region's Cancer Alliance). The aim of this pilot study was to assess the feasibility, uptake and impact of implementing a comprehensive 'opt-out' tobacco addiction treatment pathway for smokers admitted to Wythenshawe Hospital. The pilot study ran from 01 October 2018 to 31 March 2019. To allow for completion of the 12-week follow-up, data analysis was undertaken in August 2019 and consisted of a retrospective analysis of the data captured within the CURE forms in the hospital's electronic patient record (EPR) system.

\section{Setting and participants}

Wythenshawe Hospital is a major acute teaching hospital with approximately 900 beds, providing both district general hospital services and specialist tertiary services. Tertiary services include cardiology, cardiothoracic surgery, heart and lung transplantation, respiratory conditions, burns and plastics, cancer, and breast care services. All adult smokers admitted to Wythenshawe, excluding day-case and maternity admissions (which have separate smoking cessation services in place), were included in the pilot study.

\section{Support and engagement}

The ambition of the CURE project was to change the culture across a hospital system to view tobacco addiction as a physical disease and, therefore, ensure that all smokers have fair and equal access to the most effective treatments for their disease. The CURE project therefore adopted a whole-system approach involving a multidisciplinary project team, and based on the core components of National Institute for Health and Care Excellence PH48 guidance. ${ }^{10}$ The first step was to secure senior project management support for the duration of the development and implementation period and establishing a project steering group including the chief executive, senior hospital management, the CURE clinical lead (respiratory physician), the CURE specialist nurse lead (pay band 7) and representatives from pharmacy, IT development, business intelligence, the education and training team, local clinical commissioning group (CCG), local general practitioners (GPs) and our Greater Manchester collaborators.

\section{Training, protocols and workforce}

To integrate the provision of brief advice and cessation pharmacotherapy into routine clinical practice the clinical team designed, filmed and edited two new e-learning modules for the CURE project: level 1, which covered the provision of brief advice to smokers in the hospital and communicating with smokers in the hospital grounds; and level 2, which provided training on the evidence base behind pharmacotherapy for tobacco addiction and on the CURE prescribing protocol. The CURE prescribing protocol was designed to classify smokers into low, moderate and high-level addiction groups and provide a specific nicotine replacement therapy (NRT) regimen for each group, with varenicline therapy as an alternative or additional treatment for all (see supplementary material S1). ${ }^{711-13}$ The clinical lead also provided a significant number of teaching sessions throughout the hospital including grand rounds, foundation year training, core medical training teaching, core surgical training teaching and departmental/ directorate teaching as well as at GP educational events with the help of CCG colleagues. CURE posters, screensavers, banner pens and reference cards (credit card sized pathways that fit into the back of identification badge holders) were designed and disseminated across the hospital. Behavioural interventions to smokers were provided by the CURE team requiring the recruitment of three additional specialist nurses (pay band 6).

\section{Information technology}

The IT development team designed, tested and implemented an electronic screening form included in the nursing admission pro forma within the hospital's EPR system, which required smoking status to be recorded within each patient record. Answering 'Yes' to the question 'Does the patient currently smoke?' automatically flagged that patient in a CURE inpatient list within the EPR system, providing a list of smokers for the specialist team to approach on an 'opt-out' basis.

\section{Assessment and treatment}

The admitting nursing and medical team are prompted in the EPR admission clerking process to provide brief advice to quit and offer NRT/varenicline at the point of admission to a ward, and to record the outcomes of these offers. All smokers were then approached by the specialist CURE team for detailed evaluation of current and former smoking history and previous quit attempts; the provision of intensive quit advice; a discussion of all treatment options for tobacco addiction; behavioural support including motivational interviewing, brief solution focus, coping strategies for triggers and cravings and active listening; and to supply any further necessary medications either via a patient group direction (PGD) for nicotine products, or a prescription via the patient's medical team for varenicline. ${ }^{14}$ The content of this assessment is recorded within a dedicated electronic form within the EPR system, which is sent to the GP at the time of discharge as the record of this assessment and treatment plan.

\section{Discharge pathway}

At the point of discharge any patients started on tobacco addiction medications during the admission (by the medical team or by the CURE team) were provided with 7 days of medication 
to take home, with agreement and funding from the local CCG for further medications to be provided by the patient's GP for up to 12 weeks. All smokers seen by the specialist CURE team were offered follow-up at 2, 4 and 12 weeks post discharge, with the 4-week appointment offered as a face-to-face consultation with carbon monoxide validation of smoking status ( $>10$ parts per million of exhaled carbon monoxide classified as a smoker) and the 2 and 12-week follow-up being telephone consultations. At the patient's request, the 4 -week follow up could also be conducted by telephone. The 2, 4 and 12-week follow-up assessments were also recorded within dedicated EPR forms. The CURE pathway is summarised in Fig 1.

\section{Assessment of feasibility, uptake and impact}

We hypothesised that this pilot study would demonstrate that implementing the CURE project is feasible and would significantly improve the uptake and impact of tobacco addiction treatment and support at Wythenshawe Hospital in comparison to national UK audit data from the British Thoracic Society (BTS). We identified the following parameters as markers of feasibility, uptake and impact.

> Proportion of adult admissions that have electronic screening for smoking status completed (national performance: $73 \%$ of patients have smoking status recorded in medical notes).

$>$ Proportion of smokers provided with brief advice at the point of admission.
> Proportion of smokers prescribed cessation pharmacotherapy during admission (national performance: $4 \%$ prescribed NRT, $<1 \%$ prescribed varenicline).

> Proportion of smokers that complete inpatient behavioural interventions with a specialist smoking cessation practitioner (national performance: 6\% referred to a hospital smoking cessation service).

> Proportion of smokers that complete outpatient support and counselling following discharge.

> Quit rate at 4 and 12 weeks both in those completing follow-up and on an intention-to-treat basis (assuming that any smoker that did not complete follow-up continues to smoke).

> Cost per quit as a marker of cost-effectiveness / return on investment.

\section{Results}

\section{Participants}

During the 6 months of the pilot study (01 October 2018 to 31 March 2019) the hospital's EPR system recorded 14,690 adult admissions to Wythenshawe Hospital, excluding daycase and maternity admissions. Of these admissions the electronic screening of smoking status was completed in $92 \%$ $(13,515 / 14,690)$. In those patients in whom the smoking status was completed, $18 \%(2,393 / 13,515)$ were identified as current smokers.
Fig 1. CURE project pathway overview. $\mathrm{CO}=$ carbon monoxide; $\mathrm{GP}=$ general practitioner; NRT = nicotine replacement therapy; VBA = very brief advice.

\section{Active smoker admitted to hospital}

\author{
Admitting team \\ - Screen the patient for smoking status and identify as 'active smoker' \\ - Smoking status is recorded in electronic patient record / system \\ - Automated referral to the CURE team completed via electronic system \\ - Smoker is provided with brief advice \\ - Smoker is classified as 'low', 'moderate' and 'high' level addiction \\ - Smoker is offered NRT in accordance with the treatment pathway \\ and level of addiction (NRT started with 24 hours of admission) \\ - Smoker is offered varenciline in accordance with treatment pathway
}

Supported by CURE e-learning modules 1 and 2 providing training on VBA and prescribing pharmacotherapy for tobacco addiction
CURE team review: within $\mathbf{4 8}$ hours of admission

- Smoker is offered a 45 minute consultation with a specialist stop smoking practitioner

- Current NRT reviewed and amended as required

- Need for additional medications assessed (varenicline, bupropion)

- Behavioural change support and motivational interviewing

- Signposting to sources of support after discharge

- Follow-up plan agreed

- Specialist assessment sent to smoker's GP (ideally an automated process) 


\section{Interventions}

At the point of admission $96 \%(2,308 / 2,393)$ of smokers were provided with brief advice. The admitting medical team prescribed NRT for $51 \%(1,224 / 2,393)$ of smokers. Through the opt-out model, all 2,393 smokers identified via the EPR screening question were referred to the specialist CURE team, and 61\% (1,450/2,393; including $69 \%(999 / 1,450)$ of these within 48 hours of admission) accepted and completed a specialist assessment with behavioural interventions. The remaining smokers either declined the support of the specialist team or were unable to be reviewed prior to discharge. Patients who were discharged before the CURE team could see them (the CURE service was provided from Monday to Friday) were contacted by telephone where possible and offered remote behavioural interventions and pharmacotherapy advice. During the specialist assessment, the CURE team recommended 824 new medications or alterations to the medical management of tobacco addiction, with the final recommended therapies summarised in Fig 2. This included 344 additional patients being prescribed cessation pharmacotherapy that had not been prescribed any medications prior to the CURE team assessment. In total, through both the clinical teams and the CURE specialist team, $66 \%(1,568 / 2,393)$ of smokers were prescribed cessation pharmacotherapy, including $10 \%(241 / 2,393)$ that were prescribed varenicline, frequently alongside NRT.

\section{Outcomes}

Following discharge, $46 \%(1,105), 49 \%(1,179)$ and $33 \%(800)$ of all smokers admitted during the pilot completed follow-up at 2,4 and 12 weeks, respectively $(76 \%, 81 \%$ and $55 \%$ of smokers completing an inpatient CURE team assessment, respectively). At the 4-week follow-up, 495 patients reported that they had stopped smoking representing $42 \%$ of those completing followup at 4 weeks and $21 \%$ of the entire cohort of smokers admitted to Wythenshawe (assuming any patient not completing / lost

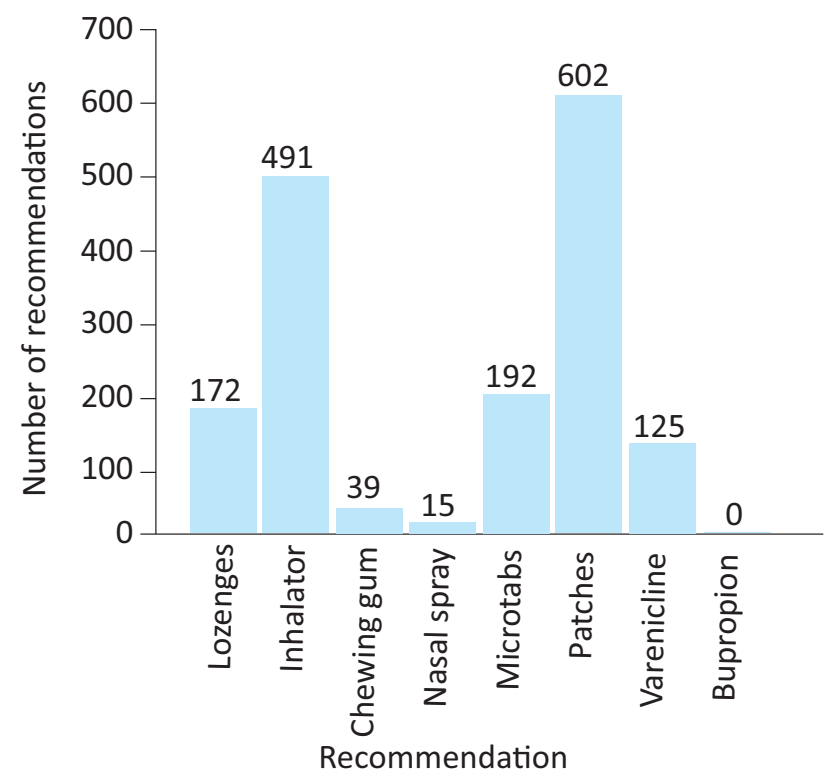

Fig 2. CURE team pharmacotherapy recommendations. to follow-up has relapsed or continued to smoke). Validation of self-reported smoking status by exhaled carbon monoxide at 4 weeks was carried out in 398 participants that agreed to attend a face-to-face consultation, and of these 293 (12\% of all smokers) recorded levels below 10 parts per million. At 12 weeks, 525 patients reported that they had stopped smoking, representing $66 \%$ of those completing follow-up at 12 weeks and $22 \%$ of the entire cohort of smokers admitted to Wythenshawe (Table 1).

\section{Costs}

Total NRT and varenicline pharmacotherapy costs for the pilot (the hospital component of treatment including the 1 week of medications post discharge) was $£ 27,974$. The staffing costs for the CURE pilot (three pay band 6 specialist nurses plus administration support) was $£ 68,250$. The total cost was $£ 96,224$ and equates to $£ 183$ per successful quit based on 525 self-reported quits at 12 weeks. Given only $59 \%$ of selfreported quitters at 4 weeks were successfully validated, a more conservative estimate is $£ 310$ /quit assuming the same proportion of validated quits would be seen at 12 weeks.

The total monthly spend on smoking cessation pharmacotherapy pre- and post launch of the CURE pilot within the South Manchester CCG from January 2018 to April 2019 is provided in Fig 3. It suggests since the launch of the CURE project there has been an approximate increase in spending on tobacco addiction pharmacotherapy of $£ 5,000-£ 7,500$ per month.

\section{Discussion}

Key results

This paper provides the first reported evaluation of the feasibility, uptake and impact of a comprehensive smoking ascertainment and treatment pilot, based on the Ottawa model, in a UK acute medical treatment setting. The results demonstrate that introducing the CURE model of systematic smoking cessation support is feasible; $92 \%$ of adult admissions were screened for smoking status and identified 2,393 smokers in whom 96\% were provided with brief advice and $66 \%$ were prescribed cessation pharmacotherapy. The pilot study demonstrated excellent uptake; $61 \%$ of smokers completed inpatient counselling and behavioural interventions with a specialist cessation practitioner, $49 \%$ completed follow-up at 4 weeks and $33 \%$ at 12 weeks. Finally, the CURE pilot was impactful; $22 \%$ of smokers were abstinent at 3 months following discharge (on an intention-to-treat basis) at a cost of $£ 183$ per quit. Therefore, the implementation of this programme achieved self-reported smoking cessation in more than one in five of all smokers admitted to the hospital. This figure suggests that if implemented nationally, this service model could generate more than 200,000 successful quits among the more than one million smokers admitted to hospitals in England each year. ${ }^{15}$ Since this has been achieved at a cost of $£ 183$ per successful quit, a figure almost identical to the estimated cost per quit in an acute setting predicted in 2018 by the Royal College of Physicians (RCP), the CURE programme represents a highly costeffective intervention and a significant return on investment. ${ }^{1}$ The RCP estimated that implementing comprehensive opt-out tobacco addiction treatment services across NHS secondary care would save the NHS approximately $€ 60$ million within 1 year, but this estimate was based on uptake of treatment by $27 \%$ of smokers. ${ }^{1}$ 
Table 1. Uptake and impact of the CURE pilot intervention during inpatient admission and follow-up after discharge

\begin{tabular}{|c|c|c|c|c|c|}
\hline & $\begin{array}{l}\text { Total } \\
\text { number, } \mathrm{n}\end{array}$ & $\begin{array}{l}\text { Of all } \\
\text { smokers, \% }\end{array}$ & $\begin{array}{l}\text { Number } \\
\text { of quits, } n\end{array}$ & $\begin{array}{l}\text { Quit rate, } \\
\text { \% with follow-up } \\
\text { data }\end{array}$ & $\begin{array}{l}\text { Quit rate, } \\
\% \text { of all } \\
\text { smokers }\end{array}$ \\
\hline All smokers & 2,393 & 100 & $\mathrm{n} / \mathrm{a}$ & $\mathrm{n} / \mathrm{a}$ & $\mathrm{n} / \mathrm{a}$ \\
\hline $\begin{array}{l}\text { Completion of inpatient CURE assessment and } \\
\text { treatment }\end{array}$ & 1,450 & 61 & $\mathrm{n} / \mathrm{a}$ & $\mathrm{n} / \mathrm{a}$ & $\mathrm{n} / \mathrm{a}$ \\
\hline Completion of 2-week follow-up post discharge & 1,105 & 46 & $\mathrm{n} / \mathrm{a}$ & $\mathrm{n} / \mathrm{a}$ & $\mathrm{n} / \mathrm{a}$ \\
\hline Completion of 4-week follow-up post discharge & 1,179 & 49 & 495 & 42 & 21 \\
\hline Completion of 12 -week follow-up post discharge & 800 & 33 & 525 & 66 & 22 \\
\hline
\end{tabular}

In the CURE pilot, $61 \%$ engaged with the service suggesting the savings to NHS may be substantially higher.

\section{Comparison to published evidence}

The CURE pilot results compare favourably to published data on smoking cessation interventions in hospital settings. A national audit of smoking cessation services and outcomes across UK hospitals, completed by the BTS in 2016, identified that in 14,750 hospital admissions $73 \%$ had smoking status recorded. ${ }^{5}$ This compares to $92 \%$ in the CURE pilot. The uptake of inpatient specialist counselling (61\% vs $6 \%$ ) and pharmacotherapy ( $66 \%$ vs $4 \%$ ) were also significantly higher in the CURE pilot compared with this audit. A feasibility study in South Carolina has also examined the uptake of a hospital-based opt-out tobacco addiction treatment service. ${ }^{16}$ In this 15 month long study, 42,061 adult admissions were recorded and 8,423 smokers identified $(20 \%)$. Overall $69 \%(5,843 / 8,423)$ of smokers were referred to the service and $33 \%(1,918 / 5,843)$ were approached by a cessation practitioner. Of those approached by a cessation practitioner $80 \%(1,535 / 1,918)$ accepted inpatient counselling though this represented $18 \%(1,535 / 8,423)$ of the intention-totreat population. The main limitation of this service was having a single full-time counsellor providing the intervention. The CURE pilot funded three full-time pay band 6 specialist nurses and engaged with $61 \%$ of the intention-to-treat population. The uptake of follow-up after discharge was also higher in the CURE pilot compared with the South Carolina study $(49 \%$ vs $28 \%$ at 4 weeks) as was the quit rate in the intention-to-treat population ( $22 \%$ vs $13.5 \%$ ). Murray et al performed a randomised controlled
Fig 3. Impact on smoking cessation pharmacotherapy monthly spend in South Manchester CCG.

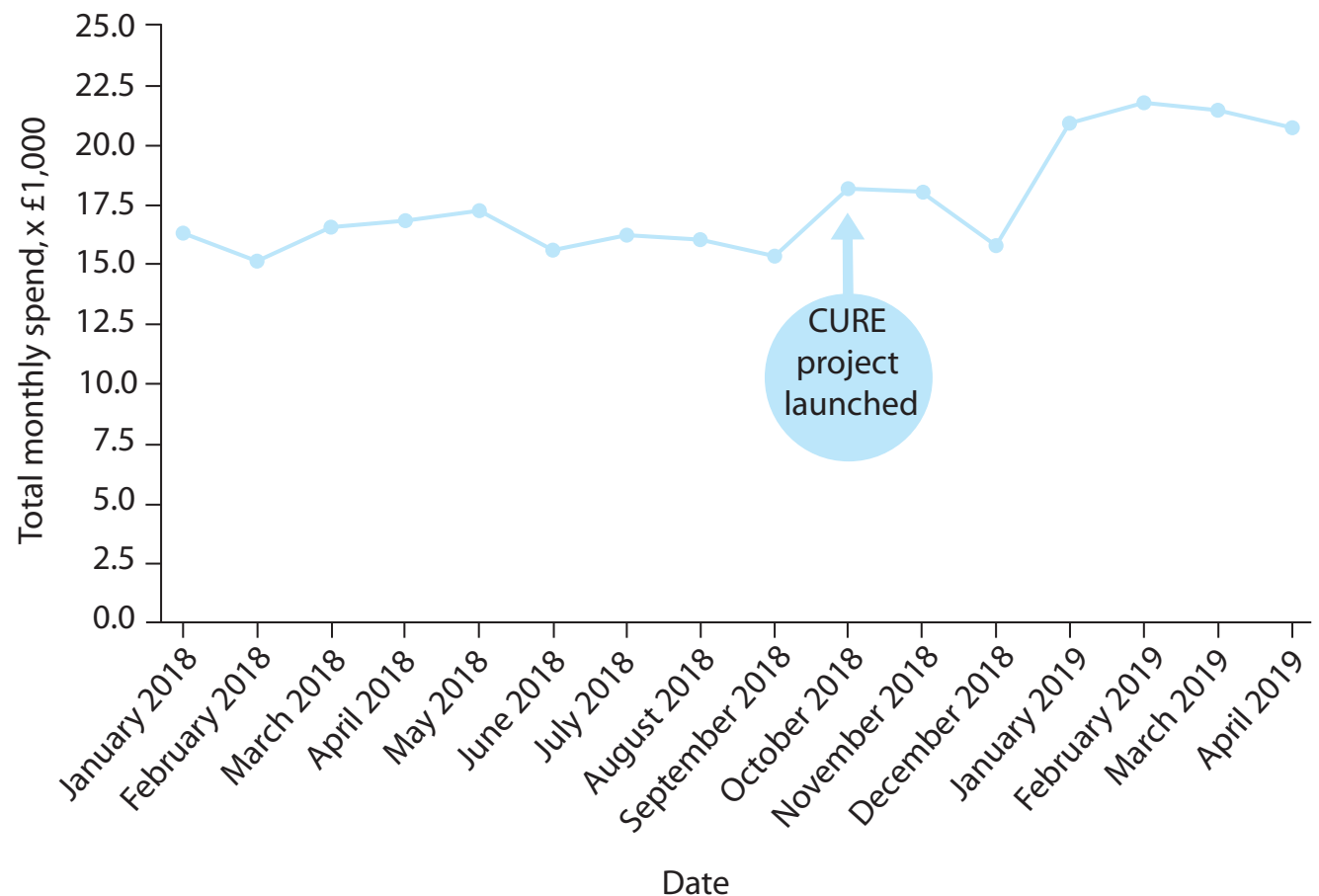


trial of a systematic opt-out offer of behavioural interventions and pharmacotherapy to inpatient smokers versus usual care across 18 medical wards of a large UK hospital. ${ }^{6}$ In the intervention group (264), the uptake of behavioural interventions with a trained practitioner was very similar to the CURE pilot (185/264; 70\% vs $61 \%)$. The uptake of both pharmacotherapy $(133 / 264 ; 50 \%$ vs $66 \%)$ and follow-up after discharge ( $80 / 264 ; 31 \%$ vs $49 \%)$ was higher in the CURE pilot compared with the intervention group of the trial. In the intervention group, the 4 -week intention-to-treat quit rate was $38 \%$, dropping to $19 \%$ at 6 months, compared with $21 \%$ at 4 weeks and $22 \%$ at 12 weeks in the CURE pilot. The lower quit rates seen in the CURE project may reflect the difference in research trial outcomes and real-life service delivery. In this randomised controlled trial, 1,072 admissions were identified as current smokers in the study period, 802 were approached and 493 randomised ( $46 \%$ ); those not entering the trial may have relevant differences to those recruited to the trial which could have an impact upon cessation success. The quit rates and uptake rates could be lower if the denominator for uptake and quit rates was all smokers admitted during the study period, as we have done in the CURE pilot results.

\section{Comparison to the Ottawa model}

The OMSC is widely referenced as an exemplar of secondary care tobacco addiction treatment and provides the foundations for the argument that replicating similar services will have significant benefits in admission savings and lives saved. ${ }^{4}$ In the Ottawa study, a control group of 641 inpatient smokers were compared with an intervention group of 726 inpatient smokers that were treated by the OMSC pathway, which consisted of a bedside inpatient review with a trained practitioner who completed a standardised form, recommended pharmacotherapy for the inpatient stay and hospital discharge, provided brief counselling and registered the patient with an automated voice recognition follow-up service. This follow-up service provided eight phone calls over 6 months, with relapse or low motivation triggering a telephone call from a practitioner. The study reported that in the control group, 6-month smoking status was available in 221 patients with 45 abstinent from tobacco $(20.4 \%)$, while in the intervention group smoking status was available in 256 patients with 90 abstinent from tobacco $(35.2 \%)^{3}{ }^{3}$ However, the total number of admissions screened in the intervention group was 4,617 with 908 eligible for the study (smokers) and 726 were recruited. Therefore, the intention-to-treat quit rate at 6 months for the OMSC intervention group, using the total number of smokers admitted in the study period as the denominator and assuming those without follow-up data have relapsed to smoking (as we have reported in the CURE pilot) was 10\% (90/908).

The intention-to-treat quit rate of $22 \%$ at 12 weeks in the CURE pilot is therefore encouraging and is favourable to published data outlined in this manuscript. There are a number of potential reasons behind this. CURE has a focus on ensuring all frontline clinicians, across the hospital workforce, have the confidence and competence to treat tobacco addiction and that our frontline staff are proactively prescribing medical therapy from the moment of admission. This is reflected in the high proportion of patients prescribed pharmacotherapy during the pilot. This has the benefit of addressing cravings and withdrawal symptoms early in the admission and potentially improving compliance with the programme and doesn't rely solely on cessation practitioner delivering the intervention. The CURE pilot provided intensive support and behavioural interventions delivered by specialist nurses (pay band 6) during the inpatient stay as well as on discharge. This level of experience and nursing expertise is likely to be an important component of the pilot's success.

\section{Limitations}

There are a number of limitations to this pilot study. There is a lack of a comparator group or robust pre-programme data to compare our outcomes against and we are therefore measuring our impact against previous national audit data and published trial data. This audit and published data may not be reflective of Wythenshawe outcomes prior to CURE. Our electronic screening question for smoking status uses a very simplistic question based on selfreported status. This simplicity is helpful and efficient for our frontline staff, however, it could potentially lead to some smokers being missed. Lack of time or reluctance to discuss smoking status could potentially lead to patients reporting as an ex-smoker that may have smoked within the days preceding admission being missed by the service. Finally, we do not know if these results are replicable to other hospitals and whether the same results can be achieved. This pilot study utilised band 6 specialist nurses to deliver the service but this may not be replicable at scale and it is not known what impact a different workforce may have on outcomes. In 2020, the CURE project is launching in six additional acute hospital trusts in Greater Manchester which will yield further data about the feasibility of wider roll out and the impact of different skill sets delivering CURE.

\section{Conclusions}

We have successfully piloted a new comprehensive tobacco addiction pathway that has led to over one in five of all smokers admitted to the hospital successfully stopping smoking, and initiating a widespread change in treatment culture in relation to smoking across the hospital. The CURE pilot provides a potential model for implementation of national secondary care cessation services as outlined in The NHS Long Term Plan. ${ }^{17}$

\section{Supplementary material}

Additional supplementary material may be found in the online version of this article at www.rcpjournals.org/content/clinmedicine: S1 - CURE project prescribing protocol.

\section{Funding and acknowledgements}

The funding to develop the CURE e-learning modules was provided by Wythenshawe Hospital charitable funds. Funding for the pharmacotherapy and staff for the CURE pilot was provided by the Make Smoking History team, the tobacco control arm of the Population Health Board, Greater Manchester Health and Social Care Partnership. Additional funding to accelerate the implementation of CURE though ongoing project management and communications strategy was provided through a joint working contract between Pfizer and NHS England on behalf of Greater Manchester Health and Social Care Partnership Board, details of which can be found on the websites of both organisations. Kelly Shennan, medical illustration team at Wythenshawe Hospital, designed the branding and communications of the CURE project. 


\section{References}

1 Royal College of Physicians. Hiding in plain sight: Treating tobacco dependency in the NHS. London: RCP, 2018.

2 Critchley JA, Capewell S, Unal B. Life-years gained from coronary heart disease mortality reduction in Scotland: prevention or treatment? J Clin Epidemiol 2003;56:583-90.

3 Mullen KA, Manuel DG, Hawken S] et al. Effectiveness of a hospitalinitiated smoking cessation programme: 2-year health and healthcare outcomes. Tob Control 2017;26:293-9.

4 Evison M, Agrawal S, Conroy M et al. Building the case for comprehensive hospital-based tobacco addiction services: Applying the Ottawa model to the City of Manchester. Lung Cancer 2018;121:99-100.

5 Agrawal S, Mangera Z. British Thoracic Society smoking cessation audit report: Smoking cessation policy and practice in NHS hospitals: National audit period: 1 April - 31 May 2016. British Thoracic Society, 2016

6 Murray RL, Leonardi-Bee J, Marsh ] et al. Systematic identification and treatment of smokers by hospital based cessation practitioners in a secondary care setting: cluster randomised controlled trial. BM] 2013;347:f4004.

7 Windle SB, Filion KB, Mancini JG et al. Combination therapies for smoking cessation: a hierarchical Bayesian meta-analysis. Am J Prev Med 2016;51:1060-71.

8 Cahill K, Stevens S, Perera R, Lancaster T. Pharmacological interventions for smoking cessation: an overview and network meta-analysis. Cochrane Database Syst Rev 2013:CD009329.

9 Rigotti NA, Clair C, Munafo MR, Stead LF. Interventions for smoking cessation in hospitalised patients. Cochrane Database Syst Rev 2012:CD001837.
10 National Institute for Health and Care Excellence. Smoking: acute, maternity and mental health services: Public health guideline [PH48]. NICE, 2013.

11 Anthenelli RM, Benowitz NL, West R et al. Neuropsychiatric safety and efficacy of varenicline, bupropion, and nicotine patch in smokers with and without psychiatric disorders (EAGLES): a double-blind, randomised, placebo-controlled clinical trial. Lancet 2016;387:2507-20.

12 National Institute for Health and Care Excellence. Varenicline for smoking cessation: Technology appraisal guidance [TA123]. NICE, 2007.

13 Mills EJ, Wu P, Lockhart I, Thorlund K, Puhan M, Ebbert JO. Comparisons of high-dose and combination nicotine replacement therapy, varenicline, and bupropion for smoking cessation: a systematic review and multiple treatment meta-analysis. Ann Med 2012;44:588-97.

14 Lancaster T, Stead LF. Individual behavioural counselling for smoking cessation. Cochrane Database Syst Rev 2017;3: CD001292.

15 Szatkowski L, Murray R, Hubbard R, Agrawal S, Huang Y, Britton J. Prevalence of smoking among patients treated in NHS hospitals in England in 2010/2011: a national audit. Thorax 2015;70:498-500.

16 Nahhas GJ, Wilson D, Talbot $V$ et al. Feasibility of implementing a hospital-based 'opt-out' tobacco-cessation service. Nicotine Tob Res 2017:19:937-43.

17 NHS England. The NHS Long Term Plan. NHS, 2019.

Address for correspondence: Dr Matthew Evison,

Wythenshawe Hospital, Manchester University NHS

Foundation Trust, Southmoor Road, Manchester, M23 9LT, UK.

Email:m.evison@nhs.net Treating tobacco dependency in the NHS

Published in 2018, this report by the Tobacco Advisory Group of the Royal College of Physicians addresses the harms and costs arising from smoking in the patients we see every day, and argues for a new approach to treating their addiction.

Download the report at: www.rcplondon.ac.uk/hidingplain-sight-treating-tobaccodependency-nhs

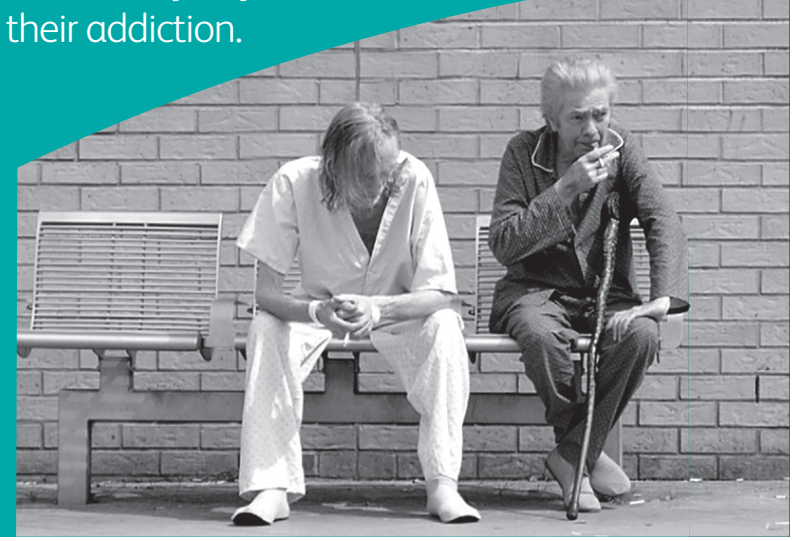

